Planetary Systems in the Universe - Observation, Formation and Evolution

Proceedings IAU Symposium No. 202, (C)2004 IAU

Alan Penny, Pawel Artymowicz, Anne-Marie Lagrange, \& Sara Russell, eds.

\title{
The Search for Terrestrial Planets: What Do we Need to Know?
}

\author{
Charles Beichman \\ Jet Propulsion Laboratory, California Institute of Technology, \\ Pasadena, CA 91109, U.S.A.
}

\begin{abstract}
The goal of finding and characterizing habitable planets in other solar systems represents one of humanity's greatest scientific challenges. NASA and ESA have initiated studies of missions that could accomplish this goal within the next ten years. What precursor knowledge do we need before we can initiate such a mission? How large should the first steps be in a program whose ultimate aim is to detect life on other planets? This talk describes different concepts for NASA's Terrestrial Planet Finder and discusses potential precursors in a program that balances scientific return, technological advance, and programmatic risk.
\end{abstract}

\section{Introduction}

The public and the scientific response to NASA's search for habitable planets and life has been very enthusiastic. Most recently, the National Academy of Science's Decadal review of Astronomy and Astrophysics (2000) endorsed the Origins goals, noting:

"Key problems that are particularly ripe for advances in the coming decade... [include] studying the formation of stars and their planetary systems, and the birth and evolution of giant and terrestrial planets."

"Search for Life outside of earth and, if it is found, determine its nature and its distribution in the galaxy... [This] is so challenging and of such importance that it could occupy astronomers for the foreseeable future."

But the NAS panel added a caveat to its recommendation that NASA proceed with the Terrestrial Planet Finder mission (TPF):

"The committee's recommendation of this mission is predicated on the assumptions that TPF will revolutionize major areas of both planetary and non-planetary science, and that, prior to the start of TPF, ground- and space-based searches will confirm the expectation that terrestrial planets are common around solar type stars."

This talk addresses what we must learn about the prevalence (or absence) of Earth-like planets before starting TPF and discusses what missions might play a role in a balanced program to advance our scientific knowledge and technological prowess to the level needed for TPF. 


\section{Finding Planets Before TPF}

As described by the TPF Science Working Group, the primary challenges of the Terrestrial Planet Finder (TPF) are to "detect radiation from Earth-like planets in the habitable zones surrounding a statistically valid sample of $\sim 150$ solar type (spectral types F, G, and K) stars, to characterize the orbital and physical properties of all detected planets to assess their habitability, and to characterize the atmospheres and search for potential biomarkers among the brightest candidates." The specified number of stars is a compromise between providing a high probability of success in finding habitable planets, setting a meaningful limit on the prevalence of planets in the event of a negative result, and the demands on the observing system.

The average distance to the stars with terrestrial planets is perhaps the most critical parameter that sets the scale of TPF. The baseline TPF concept calls for operation toward stars as far away as $\sim 15 \mathrm{pc}$, beyond which distance either the angular resolution or sensitivity become insufficient. If, however, Earths were known to be ubiquitous, then one could concentrate on the closest stars $(<5 \mathrm{pc})$ using smaller telescopes and lower angular resolution. Conversely, if Earths were known to be rare, it would be necessary to use larger telescopes to look beyond $15 \mathrm{pc}$ to find even a few examples. Thus a major goal for a precursor TPF science program is to assess the fraction of stars with terrestrial planets in the habitable zones, denoted $\eta_{\oplus}$. A lesser but still valuable goal would be to assess the fraction of systems with giant planets located only beyond the habitable zone so that Earths would have a chance to form and survive on stable orbits.

\section{1. (Almost) Everything I Know About Planets I Learned from Radial Velocities}

As described at this conference, the radial velocity (RV) technique (Mayor and Queloz 1995; Marcy and Butler 2000) has proven to be the most successful technique to date in finding planets and even planetary systems. With almost 50 planets now known (see the invaluable Extrasolar Planets Encyclopaedia at http://www.obspm.fr/planets; Schneider 1996), we have learned that approximately $5 \%$ of stars have gas giant planets located within a few AU of their parent star and ranging in size from $0.17 / \sin i \mathrm{M}_{J}$ (Marcy \& Butler, this conference; and Udry, Mayor \& Queloz 2000) to beyond the $13 \mathrm{M}_{J}$ deuterium burning limit. As many have noted, these gas giants all exist at orbital radii well within water-ice condensation line thought to be the interior limit for the accretion of such planets. Whether this discrepancy is due to dynamical effects or to an insufficient understanding of the mechanisms of planet formation is a matter of great debate.

While only two planetary systems are known, ( $v$ And and HD83443), Marcy (this conference) claims that the residuals in the RV data for half of his sample exceed the experimental uncertainties and are highly suggestive of the presence of planets with periods longer than the present observational datasets. While multiple systems may eventually prove be common, as yet we know of no counterpart to our own "grand design" solar system with gas giants on circular orbits located beyond the water-ice condensation line and rocky planets nestled within the habitable zone. Furthermore, the broad range of eccentricities and small or- 
bital radii of the known giant planets may be inconsistent with the existence of habitable, terrestrial planets. Some have argued that these unexpected results mean that solar systems like our own are rare. However, many would argue that we are simply reaping the fruits of using a technique (radial velocity) that is biased toward finding gas giant planets on short period orbits. What are the properties of the solar systems (if any) orbiting the $95 \%$ of solar type stars for which the RV technique has to date set only upper limits? To make further progress we need RV data over longer periods to probe larger orbital distances, but we also need qualitatively new types of data.

\subsection{Transit Experiments}

A second technique that has recently borne fruit is the measurement of the transit of a planet in an edge-on orbit across the face of its parent star. The detection of the transits of HD 209458 (Charbonneau et al. 2000; Henry et al. 2000) is a spectacular demonstration of the power of this technique and a validation of the planetary interpretation of the RV data. In addition to giving the planet's true mass (by determining the inclination angle), radius and density, spectroscopic observations might even reveal information the composition (Sasselov and Seager 2000). On the other hand, the failure to find any transits toward to $3.4 \times 10^{4}$ stars in the globular cluster 47 Tucanae (Gilliland et al. 2000) is an indication that not all environments are hospitable to the formation or survival of planets.

While Jupiter-sized planets present a readily observable transit signal of $1.5 \%$, Earths produce a signal that is only $\sim 10^{-4}$ in depth. However, if the $5 \times 10^{-5}$ accuracy of the recent HST observations of HD 209458 (Brown et al. 2000 , this conference) can be replicated or improved on in an imaging system, then the detection of Earths around other stars is plausible. A wide area survey of $\sim 10^{5}$ stars is required because transits are rare due to the requirement for precise alignment - 1 (10)\% for planets at $1(0.1) \mathrm{AU}$ - and infrequent due to orbital periods ranging from a few months up to once per year for a planet at 1 AU.

A number of groups are either already making such observations on the ground (Borucki et al. 2000; Howell et al. 2000) or proposing them for space missions, including Kepler in the US (Koch et al. 1998) and Eddington/COROT in Europe (Leger et al. 2000). These projects would target solar type stars typically a few 100 to 1,000 pc away in fields of 10-100 sq. deg. Since these weak signals must be measured at least three times to identify and then confirm their presence, a mission duration of at least 3-4 years is required to find planets in the habitable zone.

\subsection{Microlensing Searches}

Searches for planets by gravitational microlensing take advantage of the fact that the presence of a second mass in a lens system produces multiple images of a background star. The lensing star produces a magnification of the background star's brightness that lasts a month or longer and which can be as great as a factor of 3 to 10 . The second mass, located within a few $\mathrm{AU}$ of the central star, produces an additional magnification of a few percent up to a factor of two, lasting anywhere from a few days for a Jupiter to a few hours for an Earth (Peale 1997). A number of groups have reported detections of binary stars and, with 
less certainty, of objects of Jovian mass (Alcock et al. 2000). The frequency of these events is, unfortunately, quite low so that $\sim 10^{8}$ stars must be monitored continuously if the hours-long enhancements due to an Earth are to be detected.

Advantages of the microlensing technique include the large photometric signal, the early identification of the lensing system which can then be constantly monitored, the well defined signature of a microlensing event, and the ability to find free floating planets. The disadvantages include the great distances to the lensing star and the background stars (4 and $8 \mathrm{kpc}$, respectively), and the fact that the derived mass of the planet depends on the poorly known stellar mass. Further, most of the lensing stars will typically be $M$ dwarfs or smaller and thus not direct solar analogs. Proponents of both ground-based programs and space-based missions such as GEST (Bennett et al. 2000) have suggested that microlensing can reliably find tens of Earths per year.

\subsection{The Promise of Astrometry}

The complementary technique to radial velocities for searching for planets around nearby stars is astrometry. Astrometry is relatively unaffected by the photospheric motions that limit the RV technique to detections of $\sim$ Saturn-mass planets. Furthermore, astrometric signals grow rather than shrink with increasing orbital distance, reversing the selection bias that affects RV measurements. The scale of the astrometric measurement challenge is set by two cases: the signal of a Jupiter orbiting $5 \mathrm{AU}$ away from a G2V star 10 pc away is $\sim 500$ $\mu$ as while the astrometric signal of an Earth orbiting 1 AU away the same star is $\sim 0.3 \mu$ as. Detecting a planet of unknown period and amplitude requires a dataset extending at least as long as half the planet's orbital period. For reliable planet detection, the single measurement accuracy must be approximately half of the astrometric amplitude of the planetary signal (Brown et al. 1999).

Table 1. Table 1. Prospects for Astrometric Detection of Planets

\begin{tabular}{|l|r|r|r|r|r|r|}
\hline & $\begin{array}{r}\text { Start/End } \\
\text { Dates }\end{array}$ & $\begin{array}{r}\text { Largest } \\
\text { Orbit } \\
(\mathrm{AU})\end{array}$ & $\begin{array}{r}\text { Single } \\
\text { Meas. } \\
\text { Accur } \\
(\mu \mathrm{as})\end{array}$ & $\begin{array}{r}\text { Limit. } \\
\text { Mag. } \\
(\mathrm{mag})\end{array}$ & $\begin{array}{r}\text { Dist to } \\
\text { Detect } \\
\text { Jupiter } \\
(\mathrm{pc})\end{array}$ & $\begin{array}{r}\text { Dist to } \\
\text { Detect } \\
\text { Earth } \\
(\mathrm{pc})\end{array}$ \\
\hline Keck-I & $2003-$ & 15 & 30 & 15 & 85 & - \\
$\&$ VLT-I & 2025 & & & & 5 & - \\
FAME & $2005-2010$ & 5 & 500 & 9 & 5 & - \\
SIM & $2008-2013$ & 5 & $1-4$ & 10 & $>1,000$ & 3 \\
GAIA & $2012-2017$ & 5 & 40 & 10 & 60 & - \\
\hline
\end{tabular}

As summarized in Table 1, the two NASA projects for astrometric planet detection are the Keck Interferometer and the Space Interferometer Mission (SIM; Unwin et al. 1998). Keck-I will be capable of detecting Jupiters out to almost $100 \mathrm{pc}$ and Uranus-mass planets at $5 \mathrm{AU}$ out to $\sim 5 \mathrm{pc}$. And since it will operate for up to 25 years, Keck-I (and eventually VLT-I in the southern hemisphere) will be able to find planets on long period orbits and determine their masses. During its 5 year mission SIM will be able to detect planets of just a few Earth masses in 1-5 AU orbits around stars as far away as 10 pc. SIM 
will lower the mass limits into the range predicted for the "rocky" as opposed to "gas giant" planets.

There are two other astrometric missions worth mentioning in this context. NASA is planning to launch the Full Sky Astrometric Explorer (FAME) around 2005. ESA has recently selected the GAIA mission as a Cornerstone for a launch around 2012. It is important to recognize that these survey missions accomplish their final mission accuracies after averaging 1000 individual measurements. The single measurement accuracy which is critical for planet detection is $\sqrt{1000} \sim 30$ times worse than the mission accuracy, or 1,500 $\mu$ as for FAME and $120 \mu$ as for GAIA. While averaging of the data can improve the effective single measurement accuracy (say by a factor of 3), FAME and GAIA will find only giant planets.

\section{The Challenge of Direct Detection}

Finding habitable environments or even extant life ultimately requires the direct detection of photons from planets. The challenges include faint signals, an enormous contrast ratio between the star and the planet, the close proximity of the planet to the star ( $1 \mathrm{AU}$ corresponds to $0.1^{\prime \prime}$ at $10 \mathrm{pc}$ ), and the presence of zodiacal emission in our own and in the target solar systems. But as described in the ExNPS Roadmap, the Terrestrial Planet Finder monograph (Beichman, Woolf and Lindensmith 1999), and the recent Darwin report, a mission capable of achieving this goal is within our grasp.

A broad suite of TPF architectures is being investigated by four NASAsponsored studies involving 16 industrial concerns, 30 universities, 75 scientists, including a number of European researchers. The major concepts under investigation include nulling IR interferometers, visible light telescopes with coronagraphs or apodized pupils, and sparse aperture telescopes. The TPF project will choose 4-8 of the most promising concepts for more detailed study over the next 18 months.

\subsection{Reflected Light Systems}

Observing systems to detect the reflected light from an Earth $10 \mathrm{pc}$ away must utilize a large visible light telescope $\left(\sim 50-100 \mathrm{~m}^{2}\right.$ of collecting area) with an advanced coronagraph and/or pupil apodization along with precise wave front control $(<\lambda / 1000)$. The advantages of these systems include operation in a traditional imaging mode on a single spacecraft. The chief disadvantages arise from the extreme star:planet contrast ratio $\left(>10^{9}\right)$ which implies the need for exquisite suppression of diffracted and scattered light on a large (monolithic) telescope. Spectral features of $\mathrm{H}_{2} \mathrm{O}$ and $\mathrm{O}_{2}$ might be used to characterize planetary atmospheres and look for life. What makes visible coronagraphs interesting again after a decade of neglect is the new technology of small deformable mirrors with thousands of actuators capable of $<1 \mathrm{~nm}$ stability. The combination of a large telescope of diameter, D, a coronagraph, and a deformable mirror can, in principle at least, reject scattered and diffracted starlight in a field of a few arcseconds around a bright star (Malbet, Yu and Shao 1995). The number of actuators required is given by $[\text { Field } /(\lambda / D)]^{2}$ or roughly $\left[3^{\prime \prime} /\left(0.25^{\prime \prime} /(0.5 \mu m / 8 m)\right]^{2} \sim\right.$ 37,000 for a $3^{\prime \prime}$ field on an $8 \mathrm{~m}$ telescope. While the issues of telescope quality, 
stability, control of all sources of scattered light, etc., make the coronagraph approach very challenging, the technique is under active study.

\subsection{Thermal Infrared Systems}

Observing systems to detect thermal radiation incorporate a nulling infrared interferometer (Angel and Woolf 1997; TPF and Darwin reports) consisting of 46 telescopes, each $2 \mathrm{~m}$ (Darwin) - $3.5 \mathrm{~m}$ (TPF) in diameter, operating on separate spacecraft over baselines of 75-200 $\mathrm{m}$ to achieve the required angular resolution. The chief advantages of these systems include a relatively favorable star:planet contrast ratio $\left(10^{6}\right)$ and the presence of broad, deep absorption bands that can characterize planetary atmospheres, e.g. $\mathrm{CO}_{2}, \mathrm{H}_{2} \mathrm{O}$, or serve as signposts of life, e.g. $\mathrm{O}_{3}$ and $\mathrm{CH}_{4}$. The disadvantages of the interferometers include noise due to emission from zodiacal dust, the need for multiple spacecraft, and cryogenic operation.

\section{TPF Precursors}

Detecting gas giant planets is obviously easier than detecting Earths. For example, a $2 \mathrm{~m}$ telescope equipped with a coronagraph could detect and characterize Jupiters at visible wavelengths (the ECLIPSE mission; Trauger et al. 2000). Similarly, a smaller version of the infrared nulling interferometer could investigate the spectra of gas giant planets and even detect Earths around the closest stars. For example, the observing time, $\tau$, for a nulling interferometer to detect an Earth 10 pc away at $12 \mu \mathrm{m}$ scales roughly as follows (Beichman and Velusamy 1999):

$$
\tau \sim(2 h r) \times\left(\frac{R_{\text {Planet }}}{R_{\oplus}}\right)^{-4} \times\left(\frac{\text { Tel. Diam. }}{3.5 m}\right)^{-4} \times\left(\frac{\text { Dist. }}{10 p c}\right)^{4} \times\left(\frac{\text { Resln }}{3}\right) \times\left(\frac{S N R}{5}\right)^{2}
$$

Planet diameter, telescope diameter, and distance to the planet can all be traded against one another linearly. Thus, a scaled down version of the nulling IR interferometer, e.g. TPF-Lite using four $1.5 \mathrm{~m}$ telescopes on a fixed 20 $\mathrm{m}$ boom, could carry out spectroscopy of Jupiters and even detect an Earth around the stars within 5 pc (Woolf et al. 1998; Velusamy and Beichman 2000). This system would require relatively little new technology beyond the nulling capabilities being developed for the Keck Interferometer.

These modest-class missions, operating at either visible or infrared wavelengths, would give us valuable information on the physical properties of some of the gas giant planets already detected (Sudarsky et al. 2000), would identify new gas giants on distant orbits that would take many years to detect via astrometric or radial velocity measurements, and would give us real world experience with complex new observing techniques.

\section{What Can (Must) We Know Before Starting TPF?}

How will our scientific knowledge increase over the next decade to satisfy the requirements laid down by the NAS? Tables 2 and 3 summarize the information 
Table 2. Table 2. Summary of Ground-Based Capabilities

\begin{tabular}{|c|c|c|c|c|c|c|}
\hline Technique & $\begin{array}{l}\text { Tel. } \\
\text { Size } \\
(\mathrm{m})\end{array}$ & $\begin{array}{r}\text { Time to } \\
\text { Return } \\
\text { Data } \\
(\mathrm{yr})\end{array}$ & $\begin{array}{r}\text { Planet } \\
\text { Size }\end{array}$ & $\begin{array}{r}\text { Planet } \\
\text { Orbit } \\
(\mathrm{AU})\end{array}$ & $\begin{array}{r}\text { Dist to } \\
\text { Planet } \\
(\mathrm{pc})\end{array}$ & Spectra \\
\hline Radial Velocity & $3-10$ & Now & $>$ Saturn & $<3$ & $<50$ & None \\
\hline Astrometry & 2 & 5 & $>$ Uranus & $<15$ & $<25$ & None \\
\hline Transits & $1-2$ & Now & $<$ Uranus & $<2$ & $500-1,000$ & None \\
\hline MicroLensing & $2-3$ & Now & $\begin{array}{l}\text { Saturn } \\
\text { Earth? }\end{array}$ & $1-5$ & 4,000 & None \\
\hline $\begin{array}{l}\text { Interferom. } \\
\text { Imaging }\end{array}$ & $8-10$ & 2 & Jupiter & $<1$ & $<25$ & Yes \\
\hline
\end{tabular}

different facilities will (or could) provide toward answering the NAS concerns. Relevant portions of the on-going NASA progam include the following:

- The Keck Interferometer and the European VLT-I will use astrometry to extend the present (radial velocity) census of giant planets over wide range of orbital parameters. With these data in hand, we can use dynamical and evolutionary theories to infer a great deal about the prevalence of terrestrial planets, and the suitability of particular stars as potential TPF targets.

- Early in the next decade SIM will push the census of planets around nearby stars down to masses of terrestrial planets, highlighting good targets for TPF. Note that of all the proposed new capabilities, only SIM measures the masses, information that is essential to understanding the habitability of solar systems and of planets themselves.

- SIRTF, Keck Interferometer, and the Large Binocular Telescope (LBT) will characterize zodiacal dust clouds around nearby stars to a level of $<10$ times that of the solar system. We will be able to extrapolate the "disk luminosity function" to near Solar System levels to assess the importance of this noise source on the problem of planet detection.

But these projects do not provide all the information we might require. Projects that are not presently in the program that would be of considerable utility include the following:

- A mission to determine $\eta_{\oplus}$ from either transits or microlensing would set the distance scale for TPF and determine the required aperture size and angular resolution needed for a high probability of success in finding Earths. While the COROT mission is a good first step, a mission on the scale of Eddington or Kepler is required for robust statistics. An intensive ground-based microlensing campaign would a valuable near-term adjunct to this effort.

- An advanced coronagraphic telescope might detect and characterize giant planets around nearby stars and demonstrate the technologies needed for more capable future missions. 
Table 3. Table 3. Summary of Spaced-Based Capabilities

\begin{tabular}{|c|c|c|c|c|c|c|}
\hline $\begin{array}{l}\text { Technique } \\
\text { Project }\end{array}$ & $\begin{array}{l}\text { Tel. } \\
\text { Size } \\
(\mathrm{m})\end{array}$ & $\begin{array}{r}\text { Time to } \\
\text { Return } \\
\text { Data } \\
(\mathrm{yr})\end{array}$ & $\begin{array}{r}\text { Planet } \\
\text { Size }\end{array}$ & $\begin{array}{r}\text { Planet } \\
\text { Orbit } \\
(\mathrm{AU})\end{array}$ & $\begin{array}{r}\text { Dist to } \\
\text { Planet } \\
(\mathrm{pc})\end{array}$ & Spectra \\
\hline SIM & 0.4 & $5-10$ & $>$ Few Earth & $<5$ & $<10$ & None \\
\hline Transits & $1-2$ & $7-9$ & $\begin{array}{r}\text { Earth } \\
\text { (FGK stars) }\end{array}$ & $<2$ & 1,000 & Limited \\
\hline MicroLensing & $1-2$ & $5-7$ & $\begin{array}{r}\text { Earth } \\
\text { (M stars) }\end{array}$ & $1-5$ & 4,000 & None \\
\hline TPF-Lite & & & & & & \\
\hline Interferom. & $1-2$ & 8 & Saturn & $<5$ & $<25$ & $\begin{array}{l}\text { Atmo- } \\
\text { sphere }\end{array}$ \\
\hline & $1-2$ & 8 & Earth & $<5$ & $<5$ & None \\
\hline Coronagraph & 2 & 8 & Jupiter & $<5$ & $<10$ & $\begin{array}{l}\text { Atmo- } \\
\text { sphere }\end{array}$ \\
\hline TPF & & & & & & \\
\hline Interferom. & $\begin{array}{r}3.5 \\
6-10\end{array}$ & $10-15$ & Earth & $\begin{array}{l}<5 \\
<5\end{array}$ & $<15$ & Life \\
\hline Coronagraph & $6-10$ & $10-15$ & Earth & $<5$ & $<15$ & Life \\
\hline
\end{tabular}

- A scaled down version of a nulling IR interferometer would detect and characterize giant planets around nearby stars, detect Earths (if any) around the nearest stars, and demonstrate the technologies needed for more capable missions in the future.

From the programmatic standpoint, the problem with these intermediate missions is that they will be expensive and are likely to delay the start of TPF, particularly if we have to wait for their results. The transit, microlensing, and coronagraphic missions have all been proposed for NASA's Discovery program which has a $\sim \$ 300 \mathrm{M}$ cost cap; realistic missions might cost even more. A scaled down nulling IR interferometer could easily cost as much as SIM. Thus if we require these missions as precursors, we must accept delays in TPF and higher program costs.

Consider how we might combine the following four elements into a program:

Element 1. Initiate a Mission to Determine $\eta_{\oplus}$ (Launch in 2006). If an adequate number of stars can be observed with the requisite precision in a Discoveryclass mission, then either a transit or a microlensing experiment would bolster our confidence in the goals of TPF and set the distance scale for planet searches.

Element 2. Initiate a TPF-Lite Mission (Launch in 2008). An IR nulling system could make spectroscopic studies of already known giant planets, survey for new giant planets on distant orbits, and even survey the nearest stars for Earths. A visible light coronagraph like ECLIPSE could target Jupiters around nearby stars.

Element 3. Initiate Nominal TPF in 2007 (Launch in 2012). The present NASA plan for TPF involves an IR nulling interferometer based on technology from the SIM, NGST, and ST-3 missions. With a suitable redirection of tech- 
nology funds, this plan would support a coronagraph alternative if the results of the ongoing studies are favorable. However, this schedule would not allow us to incorporate into the design of TPF the knowledge gleaned from a transit or microlensing experiment, or from the SIM mission. The scale of TPF would have to be set by the compromise described above of wanting to observe $\sim 150$ stars out to $\sim 15 \mathrm{pc}$. TPF would however have a well characterized list of targets from radial velocity observations and astrometry using Keck-I, VLT-I, and SIM.

Element 4. Launch TPF based on knowledge of prevalence of Earths (Launch after 2015). If it is necessary to design TPF based on the known incidence of Earths and the actual configuration of (giant) planets around nearby stars, then we must wait until data are obtained from the statistical experiments (transits or microlensing) and/or results from SIM. Under this scenario, TPF could not be launched before 2015. Achieving a high degree of certainty requires that we wait a very long time!

The most aggressive approach, i.e. the present Origins plan, would be to proceed at full speed with the nominal TPF (Element 3). While one never has absolute certainty of what one will find in exploration, a TPF capable of looking for Earths around a few hundred stars out to 15 pc has a high probability of success except for the most pessimistic estimates of $\eta_{\oplus}$. A more deliberate approach would require an early measurement of $\eta_{\oplus}$ (Element 1 ), would fly a modest-scale precursor (IR interferometer and/or coronagraph, Element 2), and would finish with a very capable spectroscopic mission in the middle of the next decade (Element 4).

The decision between the two approaches should be made on the basis of technological readiness. If the development of the necessary TPF technologies proceeds smoothly, then we should move as swiftly as budgets will allow to the nominal TPF. If not, then a more deliberate approach makes sense, allowing near-term discoveries and demonstrations of complex observing systems in space. Over the next year, NASA must assess these options and in conjunction with ESA and other international partners come up with a viable program that addresses the age-old questions that have motivated this most exciting conference.

Acknowledgements. This work was carried out for National Aeronautics and Space Administration (NASA) by the Jet Propulsion Laboratory (JPL) under a contract with the California Institute of Technology.

\section{References}

Astronomy and Astrophysics in the New Millennium: The Report of the Astronomy and Astrophysics Survey Committee, 2000, National Academy Press, in press.

A Roadmap for the Exploration of Neighboring Planetary Systems (ExNPS), 1996, edited by C. A. Beichman, JPL Publication 96-22

Alcock, C. et al. 2000, ApJ, 541, 270

Angel, J.R. P. and Woolf, N. J. 1997, ApJ, 475, 373

Beichman, C. A., \& Velusamy, T. 1999, in Working on the Fringe: Optical and IR Interferometry from Ground and Space. Proceedings from ASP Conference Vol. 194. Edited by Stephen Unwin and Robert Stachnik, p.408

Bennett, D. et al. 2000, AAS/DPS meeting, 32, 32.06 
Borucki, W. J., et al. 2000, PASP, in press

Brown, R., Sozzetti, A., \& Casertano, S. 1999, "Simulations of Planet Searches with SIM," STScI Internal Report

Brown, T. M. et al. 2000, this conference

Charbonneau, D., Brown, T. M., Latham, D. W., \& Mayor, M. 2000, ApJ, 529, L45

Gilliland, R. L. et al. 2000, ApJ, 545, L47

Henry, G. W., Marcy, G. W., Butler, R. P., \& Vogt, S. S. 2000, ApJ, 529, L41

Howell, S. B., Everett, M., Davis, D. R., Weidenschilling, S. J., McGruder, C. H., III, \& Gelderman, R. 2000, AAS/DPS Meeting, 32, 32.03

Koch, D., Borucki, W., \& Webster, L. et al. 1998, SPIE, 3356, Space Telescopes and Instruments V, p. 599

Léger, A. et al. 2000, this conference

Malbet, F., Yu, J. W., \& Shao, M. 1995, PASP, 107, 386

Marcy, G., \& Butler, P. 2000, this conference

Marcy, G., \& Butler, P. 2000, PASP, 112, 137

Mayor. M., \&Queloz, D. 1995, Nature, 378, 355

Peale, S. J. 1997, Icarus, 127, 269

Schneider, J. 1996, Extrasolar Planets Encyclopaedia, http://www.obspm.fr/planets

Seager, S., \& Sasselov, D. D. 2000, ApJ, 537, 916

Sudarsky, D., Burrows, A., \& Pinto, P. 2000, ApJ, 538, 885

The Terrestrial Planet Finder (TPF): A NASA Origins Program to Search for Terrestrial Planets, 1999, edited by Beichman, C. A., Woolf, N. J., Lindensmith. C. A., JPL Report 99-3

Trauger, J. et al. 2000, AAS/DPS meeting, 32, 26.08

Udry S., Mayor M., \& Queloz D. 2000, preprint

Unwin, S. C., Turyshev, S. G., \& Shao, M. 1998, Proc. SPIE, 3350, Astronomical Interferometry, Robert D. Reasenberg; Ed. , 551

Velusamy, T., \& Beichman, C. A. 2000, IEEE conference, in press

Woolf, N. J., Angel, J. R. P., Beichman, C. A., Burge, J. H., Shao, M., \& Tenerelli, D. J. 1998, Proc. SPIE, 3350, Astronomical Interferometry, Robert D. Reasenberg, Ed., p. 683 